Théologiques

Théologiques

\title{
Deuil et individuation
}

\section{Une contribution de la psychanalyse jungienne à la réflexion eschatologique}

\section{Pierre Farmer et Yanick Farmer}

Volume 10, numéro 2, automne 2002

Théologie et psychanalyse. Que dit l'une au sujet de l'autre?

URI : https://id.erudit.org/iderudit/008886ar

DOI : https://doi.org/10.7202/008886ar

Aller au sommaire du numéro

\section{Éditeur(s)}

Faculté de théologie de l'Université de Montréal

\section{ISSN}

1188-7109 (imprimé)

1492-1413 (numérique)

Découvrir la revue

\section{Citer cet article}

Farmer, P. \& Farmer, Y. (2002). Deuil et individuation : une contribution de la psychanalyse jungienne à la réflexion eschatologique. Théologiques, 10(2), 141-157. https://doi.org/10.7202/008886ar

\section{Résumé de l'article}

Cet article entend aborder le thème du deuil et de la mort à travers l'approche du psychanalyste suisse Carl Gustav Jung. Ainsi, les deux premières parties du travail sont consacrées à la description et à la compréhension du processus de deuil, de même qu'aux étapes de la guérison psychologique nommée par Jung individuation. Par ce biais, nous cherchons à faire valoir la pertinence de la psychanalyse dans le champ philosophique, théologique et moral, tout en mesurant, dans la conclusion, les périls que font subir à la vie spirituelle de l'homme certains aspects de l'évolution culturelle de nos sociétés. 


\title{
Deuil et individuation
}

\section{Une contribution de la psychanalyse jungienne à la réflexion eschatologique}

\author{
Pierre FARMER \\ gérontologue \\ Yanick FARMER \\ docteur en philosophie \\ Université de Paris IV-Sorbonne
}

L'expérience de la mort, qui inévitablement frappe toute personne à un moment donné de son existence, est sans doute la plus troublante et la plus profonde qui soit. L'angoisse métaphysique qu'elle fait naître a été, à toutes les époques et au sein de toutes les cultures, un motif extrêmement puissant de la réflexion philosophique et théologique. En effet, la mort se présente, pour la réflexion qui s'y attache, comme une pensée limite qui, essayant de concevoir la fin de l'existence, est conduite au cœur même de la vie et de son sens.

Lorsque nous réfléchissons au thème de la mort, c'est le plus souvent à notre mort que nous pensons, mais un peu moins à celle d'autrui. C'est pourtant précisément à cette occasion, lors de la perte d'un être cher par exemple, qu'un individu peut être saisi par la mort et en retirer une véritable sagesse pour la vie. C'est pourquoi, parmi les questions théoriques qui surgissent autour du problème de la mort, nous avons choisi d'aborder la question du deuil, laquelle ne concerne pas seulement la fin de l'existence et ses conséquences métaphysiques, mais aussi la perte, la douleur, la souffrance et le déséquilibre qu'entraîne chez les vivants la perte d'un proche ${ }^{1}$. Le deuil, puisqu'il se rapporte autant à la vie qu'à la

1. D’une manière générale, le deuil survient à la suite de la disparition d'une personne. Cependant, ce phénomène peut aussi être étendu à la perte d'un animal (chien, chat, etc.) ou d'un objet (une maison incendiée par exemple) ayant une valeur particulière pour l'individu. 
mort, permet de joindre à la réflexion proprement morale une réflexion psychologique sur les causes et le sens de la souffrance. Dans le contexte du deuil et de la perte, l'individu doit bien souvent faire face, au-delà de l'expression spontanée de sa tristesse, aux manifestations parfois jusquelà inconscientes de souvenirs ou d'événements qui ont tissé sa vie psychologique intime. Il nous est donc paru pertinent et intéressant d'enrichir la réflexion philosophique et théologique d'un point de vue psychanalytique. Pour ce faire, nous avons choisi l'approche jungienne (du nom du célèbre psychanalyste suisse Carl Gustav Jung), par intérêt d'abord, mais aussi parce qu'elle est probablement celle qui s'est le plus volontiers autorisée à élargir l'investigation psychanalytique par des considérations théologiques et philosophiques.

Ainsi, à travers l'exploration du processus du deuil, et par l'émergence d'une réflexion corollaire sur le sens de la vie humaine, la psychanalyse vient croiser la théologie et la philosophie. Non pas pour les destituer de leur rang dans le champ de la réflexion eschatologique, mais simplement pour apporter un éclairage psychologique à un thème spirituel séculaire.

Dans le premier volet de notre exploration, nous examinerons les mécanismes de transfert qui sont plus spécifiquement impliqués dans le phénomène du deuil. Cette étape nous permettra de comprendre les sources de l'investissement affectif qui sous-tend le rapport à autrui, et qui, après la perte, est cause de la souffrance qui s'exprime lors du deuil. Au fil de cette première partie, nous verrons que la perte qui entraîne le deuil amène un déséquilibre psychique qui ne peut être surmonté que par un processus d'individuation, à savoir par un cheminement personnel au terme duquel l'individu se transforme par l'intégration de contenus inconscients refoulés qui trouvent alors à s'exprimer. La deuxième partie, quant à elle, se concentrera plus spécifiquement sur les étapes conduisant à l'individuation. Nous tenterons aussi de faire ressortir l'idée que le processus du deuil, même s'il confronte l'individu à une situation pénible, peut être globalement positif, en menant à terme la maturation personnelle de celui-ci. Et en conclusion, nous soulèverons la question de savoir si l'environnement culturel actuel des sociétés occidentales n'est pas propice à faire éviter ce genre de cheminement en maintenant l'homme dans l'illusion aveugle de sa domination technique de la nature. 


\section{Le deuil et le transfert}

La psychanalyse a toujours mis en évidence l'importance vitale du transfert pour la vie psychique de l'individu. Ainsi que le souligne Jung, le transfert ne se limite pas à la cure psychanalytique, il s'étend à l'ensemble des relations d'un individu avec le monde extérieur: "Le transfert est un phénomène naturel en soi, qui ne se produit nullement dans le seul cabinet médical, mais que l'on observe partout $[\ldots]^{2}$.» Le transfert consiste pour l'essentiel en une projection de contenus subjectifs sur un objet, ce qui a pour effet de le colorer de teintes subjectives que l'on croit lui appartenir en propre ${ }^{3}$. On pourrait traduire ce phénomène en d'autres termes, en le définissant comme une subjectivisation du réel qui pose des repères en vue de l'adaptation au monde extérieur.

Le deuil causé par la perte d'un être cher représente un cas particulier, émotionnellement très intense, de transfert. Et le défi psychologique qui se pose à l'individu lors d'une telle épreuve ne peut être que celui de l'individuation, sans laquelle le vide laissé par la perte ne sera jamais véritablement comblé. Du point de vue de l'économie psychique, le deuil provoque la rupture du lien affectif concret, mais aussi, en quelque sorte, le retournement de la libido investie, qui ne trouve plus d'objet pour s'écouler. Quand survient la mort ou la perte, c'est bien sûr d'abord la personne ou l'objet qui disparaît, avec ses qualités propres. Mais sous un autre aspect, ce n'est pas seulement l' "autre » que la personne endeuillée perd, mais aussi - et peut-être devrions-nous dire surtout —une partie de l'espace intérieur qui vivait en l'autre. En ce sens, la mort objective de l'autre s'accompagne de la mort intérieure de l'autre en nous.

La tristesse, la douleur et l'angoisse, bref la détresse créée par la perte doit être associée à la nigredo, au chaos psychologique décrit par Jung dans la Psychologie du transfert. Le lien affectif entre le sujet et autrui que favorise le transfert, et que brise la mort, est accompagné d'un écoulement progressif de la libido, qui va alors du sujet vers le monde extérieur. Évidemment, progression ici ne rime pas nécessairement avec individuation, car dans certains cas, elle peut cacher une aliénation du

2. C.G. Jung, Psychologie du transfert / trad. par É. Perrot, Paris, Albin Michel, 1980 (allemand 1946), p. 84.

3. La définition jungienne du transfert est tout à fait semblable à celle de Freud. Voir par exemple S. Freud, Introduction à la psychanalyse / trad. par S. Jankélévitch, Paris, Payot, 1982 (allemand 1917), p. 408-424. 
sujet au profit de valeurs qu'il ne maîtrise pas. Quoi qu'il en soit, à partir du moment où la mort survient, avec l'enclenchement du processus de deuil, une partie de l'équilibre psychique qui était maintenu par la relation avec l'autre se rompt. Cette situation entraîne un refoulement de la libido qui ne trouve plus à se projeter sur la personne disparue. Cette désunion est le point de départ, la première étape d'un long processus qui peut mener à l'individuation, c'est-à-dire à la réalisation d'une unité qui correspond à une nouvelle naissance. "C'est donc un état d'absence de liberté, de guerre intestine, de désagrégation, d'écartèlement entre des directions opposées, et par conséquent un douloureux état de captivité qui aspire à l'unification, à la réconciliation, à la délivrance, à la guérison, c'est-à-dire à la totalité4. »

L'expérience du suivi psychologique des personnes endeuillées enseigne qu'il existe un danger, suite au refoulement de la libido, d'exposition aux démons intérieurs que réveille la régression. En effet, selon les travaux du médecin américain Elisabeth Kübler-Ross - qui font autorité en ce domaine - , le processus du mourir se déroule en cinq étapes distinctes qui s'expriment par le biais de symptômes psychologiques précis. Ces étapes concernent d'abord la personne mourante, mais elles sont analogues à celles que peut vivre la personne endeuillée ${ }^{5}$. Jusqu'à ce que l'individu ait accepté la situation à laquelle il est confronté, il doit faire face à des phases intenses de régression de la libido qui prennent des formes diverses.

Un examen sommaire de ces «comportements régressifs » révèle une tendance au repli sur soi, parfois morbide, causée par l'irrigation par une libido régressive de contenus archaïques inconscients. Il peut s'agir entre

4. Jung, Psychologie du transfert, p. 66.

5. Kübler-Ross décrit ces étapes de la manière suivante: la première étape est celle de la dénégation (denial) et consiste en une période plus ou moins longue de refus de la situation; la deuxième étape est celle de la colère (anger) face à ce que l'on considère comme une injustice ("pourquoi moi ?"); la troisième étape est celle du marchandage (bargaining) et elle vise à vouloir allonger la vie par une série d'actions concrètes; la quatrième étape consiste en une phase de dépression (depression) qui se traduit le plus souvent par un retour sur le passé où s'expriment parfois des regrets ayant rapport à des erreurs commises ou des désirs non réalisés; finalement, la cinquième étape est celle de l'acceptation (acceptance), laquelle traduit une forme de détachement du sujet face aux événements. Pour de plus amples détails sur ce processus, voir E. KüBler-Ross, On Death and Dying, New York, Macmillan Publishing, 1969, p. 38-138. 
autres des souvenirs qui traduisent des conflits non résolus et qui de ce fait engendrent de vifs sentiments de regret ou d'amertume. Parmi ces autres comportements typiques, il y a l'inflation grossière de la personnalité, laquelle peut se manifester par le désir de n'en faire qu'à sa tête et de ne désormais écouter d'autre que soi. En tous les cas, la régression entraîne une forme plus ou moins profonde de dépression et de culpabilité attribuée à soi ou aux autres; elle peut même dans certains cas provoquer un état pathologique extrême, qui pousse le sujet vers des penchants suicidaires, vers l'obsession irréaliste et déraisonnable de la mort.

Pour faire ou refaire le chemin vers une unité intérieure, la personne endeuillée doit d'abord "faire face » à la situation, c'est-à-dire reconnaître la part d'elle-même qu'elle perd avec l'autre, en vue de récupérer ce qui avait jadis été investi dans cette personne ${ }^{6}$. À cette étape, la présence d'une personne objective, remplie d'empathie et de compassion, peut s'avérer décisive dans la mesure où elle facilite la compréhension, de même que la mise en place d'un climat psychologique empreint de confiance et de sérénité.

La reconnaissance par le sujet de la nécessité de faire «la part des choses » l'incitera à faire un retour sur soi qui peut évoquer de pénibles souvenirs, parfois sous la forme de complexes dotés d'une forte dose énergétique, et par conséquent d'une forte charge affective. Cette étape se révèle toutefois essentielle à ce que la psychanalyse jungienne appelle la conjonction, étape de renaissance et d'individuation. Elle est symboliquement représentée dans la Psychologie du transfert par l'«immersion dans le bain ${ }^{7}$ ", qui signifie une descente vers l'inconscient pour y réaliser une union avec la partie obscure de la personnalité - l'ombre - qui était projetée sur l'autre. Dans bien des cas cependant, il peut arriver que ce retour sur soi soit entravé par le déni, par le refus obstiné d'accepter les faits, ce qui engendre une forme spécialement violente de refoulement. Le refus par la personne endeuillée de laisser vivre sa douleur en la refoulant produit une vive tension inconsciente qui ne peut qu'aggraver le déséquilibre psychologique qui fait suite à la perte de l'être cher. L'émo-

6. Cette étape correspond à la "vérité nue» dans le processus de transfert. Voir JunG, Psychologie du transfert, p. 98-102.

7. Jung, Psychologie du transfert, p. 103-109. 
tion étouffée accroît la force «constellante» des complexes, et puisque "l'ombre est l'obstacle qui nous sépare le plus efficacement de la voix divine", elle contribue à ralentir la réhabilitation du sujet ${ }^{8}$.

\section{Le deuil et l'individuation: pour un retour à l'équilibre psychique}

Toute renaissance, explique Jung dans la Psychologie du transfert, débute par une stase de la libido qui provoque chez le sujet une dépression, une démotivation et une apathie face à la vie. Cette phase est imagée par les figures de la «conjonction" et de la «mort», qui décrivent l'état de stagnation qui précède la nouvelle naissance?. Pour que celle-ci soit possible, il faut renouveler l'intérêt pour une nouvelle conjonction des opposés. Il faut en d'autres mots que le moi reprenne contact avec sa base inconsciente afin de rétablir les voies pour une nouvelle progression de la libido. Pour cela, il s'agit, pour reprendre les termes de Freud, de rechercher les bases du refoulement d'une part, et de supprimer la résistance qui maintient ce refoulement d'autre part ${ }^{10}$.

La désagrégation des éléments signifie la dissociation et la dissolution de la conscience du moi, telle qu'elle existait jusque-là. L'analogie avec un état schizophrénique est évidente et doit être prise au sérieux, dans la mesure où c'est à ce moment-là, lorsque l'inconscient collectif, le non-moi psychique, vient à la conscience, que les psychoses latentes peuvent devenir aiguës. Cette période, souvent longue, de désintégration et en même temps de désorientation de la conscience, est parmi les passages les plus difficiles du traitement analytique et met parfois à très rude épreuve la patience, le courage et la confiance en Dieu tant du médecin que du patient ${ }^{11}$.

8. Cette notion de «force constellante» s'explique assez bien par le schéma hydraulique développé par l'éthologue Konrad Lorenz pour décrire l'action des instincts. Lorsqu'un instinct ne trouve plus d'objet sur lequel exercer son action, il s'ensuit une accumulation de l'énergie qui lui est propre. Cette situation a pour conséquence une augmentation interne de la motivation liée à cet instinct, de même qu'une baisse de la sélectivité face aux stimuli qui l'actionnent. En conséquence, l'instinct monopolise la vie psychique de l'individu, comme une véritable obsession, en agissant même sur des stimuli qui ne lui sont pas naturellement destinés.

9. Jung, Psychologie du transfert, p. 110-131.

10. Freud, Introduction à la psychanalyse, p. 414.

11. Jung, Psychologie du transfert, p. 133-134. 
Le but ultime de la renaissance sera la réalisation du Soi, de la personnalité globale, ce qui représente à notre avis le seul moyen durable et pleinement satisfaisant de surmonter le traumatisme provoqué par le deuil. Étant donné que, pour le moi, la fonction de relation avec l'inconscient est assurée par le complexe anima-animus ${ }^{12}$, on comprendra pour-

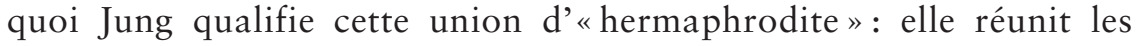
opposés psychiques au sein d'une unité qui est le Soi. "L'union du conscient ou de la personnalité du moi avec l'inconscient personnifié comme anima engendre une personnalité nouvelle qui embrasse les deux composantes "afin qu'après avoir été deux, ils deviennent en quelque sorte un seul corps" (ut duo qui fuerant, unum quasi corpore fiant). La personnalité nouvelle n'est nullement quelque chose d'intermédiaire entre le conscient et l'inconscient, elle est les deux. Comme elle transcende la conscience, elle ne doit plus être désignée comme moi, mais comme So $i^{13}$.»

Le renouveau psychique de la personne endeuillée qui commence après la stase de la libido s'érige d'abord sur une appréhension intellectuelle des contenus bruts de l'inconscient. Dans Psychologie du transfert, cette étape correspond à la " purification ${ }^{14}$ ", et, dans Mysterium conjunctionis, à la première phase de la conjonction ${ }^{15}$. Mais c'est probablement

12. L'anima et l'animus ont une place bien particulière et très importante dans la théorie jungienne de l'inconscient. Chez Jung, l'inconscient est structuré en deux niveaux. Il y a premièrement l'inconscient collectif, qui comprend l'ensemble des mécanismes innés, d'origine génétique, servant à réguler les différents processus d'apprentissage et d'interaction avec le milieu. Les structures régulatrices qui composent l'inconscient collectif se nomment archétypes. La deuxième strate est l'inconscient personnel, qui comprend l'ensemble des contenus subconscients, produits de l'interaction avec le monde extérieur: les souvenirs, les refoulements, les oublis, etc. Pour Jung, la partie refoulée de l'inconscient personnel est complémentaire de l'attitude consciente, en sorte que, par exemple, une attitude masculine engendrera un refoulement des contenus psychiques "féminins". L'anima est, chez l'homme, l'ensemble des contenus inconscients refoulés à cause de leur incompatibilité avec l'attitude consciente. L'animus est tout simplement l'équivalent de l'anima chez la femme. L'intégration de l'anima et de l'animus constitue une étape absolument essentielle à l'individuation.

13. Jung, Psychologie du transfert, p. 130.

14. Jung, Psychologie du transfert, p. 139-149.

15. C.G. Jung, Mysterium conjunctionis, t. 2 / trad. par É. Perrot, Paris, Albin Michel, 1982 (allemand 1955-56), p. 262-271. 
dans la Dialectique du moi et de l'inconscient que l'on trouve la description la plus détaillée et la plus concrète de cette phase de compréhension des contenus inconscients ${ }^{16}$. Pour que le sujet puisse éloigner ses souffrances, il doit d'abord opérer un retrait des projections, avec comme objectif de récupérer ce qu'il a investi dans la personne décédée. La compréhension des contenus inconscients et des divers éléments dont est formé le complexe nécessite une participation active du sujet. Elle exige que celuici dirige son attention et se laisse imprégner par le déroulement de ses fantasmes. De cette manière, la personne sera en mesure de saisir les motifs qui se cachent derrière ces images, ce qui aura pour conséquence de les désactiver et d'enlever aux complexes leur charge affective incontrôlable.

Même si la connaissance de soi et l'effort de compréhension intellectuelle sont une étape essentielle, elle n'est cependant pas suffisante. La confrontation conscient-inconscient exige une participation plus globale de la personnalité. Comme le précise Jung, le sujet ne doit pas se contenter d'être le spectateur de ses représentations inconscientes, il doit aussi en être l'acteur.

Mais ce qui est d'une importance essentielle, c'est que le [sujet] vive ses fantasmes de façon aussi totale que possible, et par conséquent aussi dans la mesure où une compréhension intellectuelle fait partie de la totalité du vécu - qu'il les comprenne. Mais ce n'est pas forcément la compréhension intellectuelle qu'il faut situer en haut de la hiérarchie des importances primordiales. [...] Car l'essentiel n'est pas en premier lieu l'interprétation et la compréhension des fantasmes; cela est important certes, mais l'essentiel est d'en acquérir mentalement une expérience vivante ${ }^{17}$.

Du point de vue de la conscience, l'unio mentalis - l'union intérieure - s'effectue par une participation égale des fonctions rationnelles, à savoir par un usage équilibré de la pensée et du sentiment ${ }^{18}$. L'usage de

16. C.G. Jung, Dialectique du moi et de l'inconscient / trad. par R. Cahen, Paris, Gallimard (Idées), 1964 (allemand 1928), p. 193-222.

17. Jung, Dialectique du moi et de l'inconscient, p. 195.

18. Pour ceux qui ne sont pas familiers avec l'œuvre de Jung, précisons simplement qu'il existe pour lui quatre fonctions mentales principales: la pensée, le sentiment, la sensation et l'intuition. La pensée réfère essentiellement aux lois logiques du raisonnement, alors que le sentiment est une fonction d'évaluation subjective et morale. 
fonctions qui sont par nature opposées dépend d'une considération attentive de l'anima ${ }^{19}$. Cette dernière servant de fonction d'accès à l'inconscient, elle permet d'intégrer la fonction opposée: "Ce savoir est la condition indispensable de toute intégration, ce qui veut dire qu'un contenu ne peut être intégré que lorsque son double aspect est devenu conscient, et qu'il n'est pas seulement saisi intellectuellement, mais, de plus, conforme à sa valeur affective, doit, bon gré mal gré, se confronter avec l'anima ou l'animus pour préparer une unification supérieure ${ }^{20}$ ». À son tour, l'intégration de l'anima appelle à la réalisation de l'intuition qui, en tant que fonction de perception interne, prend en considération les données inconscientes dans le processus de délibération consciente. C'est uniquement à partir de ce moment que se réalise l'union intérieure et que le sujet s'ouvre authentiquement à lui-même. En tenant compte de la fonction opposée, la nature affective et pulsionnelle des complexes est intégrée, ce qui concrétise l'union de l'esprit avec le corps ${ }^{21}$.

Pour Jung, l'intuition est une fonction de perception des données inconscientes qui, de ce fait, autorise un jugement global sur la valeur d'une représentation ou d'une situation pour l'avenir d'un individu. Face à une situation donnée, l'intuition active les mémoires inconscientes en vue de la résolution d'un problème. Ce processus extrêmement vaste, qui déborde largement le cadre de la conscience, est déterminé en partie par la configuration d'une situation. Ainsi, les contours définis par celle-ci (ce peut être entre autres la structure spatio-temporelle) détermineront dans quels registres mémoriels le cerveau puisera les solutions au problème qui

Ces deux fonctions sont dites rationnelles car elles sont produites par la réflexion consciente. La sensation et l'intuition réfèrent quant à elles à des mécanismes internes et externes de perception. Ces fonctions sont dites irrationnelles car elles sont pré-réflexives et immédiates. Pour une définition précise des différentes fonctions psychologiques, voir C.G. Jung, Types psychologiques ( $7^{\mathrm{e}}$ édition) / trad. par Y. Le Lay, Genève, Georg éditeur S.A., 1991 (1950; allemand 1921).

19. Pour un sujet féminin ce rôle sera joué par l'animus, à la différence toutefois que l'animus ne sera pas utilisé comme fonction de relation, mais plutôt comme fonction de discrimination.

20. C.G. Jung, Ä̈on / trad. par E. Perrot et M.-M. Louzier-Sahler, Paris, Albin Michel, 1983 (allemand 1951), p. 45.

21. Il ne faut cependant jamais perdre de vue les risques d'inflation du moi qui peuvent survenir suite à la confrontation avec l'inconscient. La figure-type de cette situation est la personnalité mana dont la psychologie est exposée dans Jung, Dialectique $d u$ moi et de l'inconscient, p. 223-248. 
se présente. Cependant, ce processus n'est pas purement intellectuel, au sens où il ne fait pas uniquement appel aux fonctions supérieures du cerveau. La constitution des registres et de l'univers mental d'un individu est aussi nettement influencée par son caractère, lequel est en lien direct avec les fonctions inférieures attachées aux régions subcorticales. Étant donné que pour Jung les fondements inconscients de la personnalité sont au moins partiellement d'origine génétique — comme le reste du phénotype - , l'intuition est conçue comme une fonction qui projette la pensée dans l'avenir en établissant une relation entre la situation présente et l'essence intime du caractère individuel.

L'ouverture à soi-même qui s'accomplit dans cette phase d'introspection s'avère être une étape essentielle à une nouvelle progression de la libido. Cette ouverture confronte le moi et l'inconscient, ce qui rend possible l'irruption soudaine de contenus inconscients, arrivant par l'intermédiaire de ce que Jung nomme la fonction transcendante. Cette idée, par son côté mystique, démarque nettement la psychanalyse jungienne de l'orthodoxie psychanalytique. On aurait cependant tort de la rejeter pour cette seule raison. La notion de fonction transcendante veut simplement signifier qu'à travers l'intuition, l'individu peut avoir accès au sens véritable de son existence, lequel renvoie à l'évolution de la vie personnelle de l'individu (à travers les différents registres mémoriels), comme à celle de ses ancêtres (à travers la mémoire génétique).

L'unio mentalis décrite par Jung constitue à nos yeux une étape décisive d'un nouvel équilibre pour la personne endeuillée, mais elle n'est encore qu'une étape dans un processus devant conduire à l'individuation. L'expérience douloureuse de la mort, le sentiment d'urgence qu'elle fait naître, de même qu'une sensibilité toute particulière pour ce qui vient «après » la vie, contribuent à façonner les perspectives de la personne endeuillée. À tel point que celle-ci nous paraît souvent particulièrement apte à accéder au troisième stade de la conjonction: l'unus mundus.

Le troisième et suprême degré signifie [...] l'union de l'homme intégral avec l'unus mundus. Par ce dernier terme il [faut entendre], comme nous l'avons $\mathrm{vu}$, le monde potentiel du premier jour de la création où rien n'existait encore in actu, c'est-à-dire dans le Deux et la pluralité, mais seulement dans le Un. L'unité de l'homme réalisée par un procédé magique signifie [...] la possibilité de produire également l'unité avec le monde, non pas avec la réalité multiple que nous voyons, mais avec un «monde potentiel» qui correspond au fondement éternel de toute existence empirique, tout 
comme le Soi est le fondement et la source originelle de la personnalité et comprend cette dernière dans le passé, le présent et l'avenir ${ }^{22}$.

On retrouve dans la troisième étape de la conjonction une conception du Soi qui dépasse celle d'une totalité conscient-inconscient limitée au corps physique. Ici le Soi devient une totalité cosmique reliée au corps individuel par les archétypes ${ }^{23}$. L'unus mundus signifie la réalisation complète du Soi, ce qui implique une accession de l'individu à la dimension éternelle de son existence. C'est-à-dire que dans la mesure où le Soi est perçu comme une totalité cosmique, il fait de l'homme un microcosme, un miroir du reste de l'univers. Vu sous cet aspect, le Soi inclut aussi le temps dans toutes ses dimensions (le passé, le présent et l'avenir), puisqu'il représente en quelque sorte la loi de développement de la vie d'un individu. Par conséquent le Soi établit les régularités à travers lesquelles il a été et sera possible de reconnaître le destin d'une personne. Si l'inconscient collectif, par sa structure archétypale, est le reflet de l'interaction phylogénétique entre un organisme vivant et l'environnement physique dans lequel il évolue, c'est donc qu'existe pour la conscience individuelle la possibilité de faire l'expérience d'une certaine forme de sens éternel. En fait, pour Jung, l'expérience du Soi équivaut au sentiment mystique d'une connexion intime entre Dieu et les hommes.

À l'intérieur de la conscience individuelle, l'expérience de l'éternité relie l'homme à la totalité et ne peut être vécue que grâce à un contenu mental très spécial qui tient autant compte des perceptions externes (la sensation) que des perceptions internes (intuition), et qui, pour la compréhension rationnelle, fait autant appel au sentiment qu'à la pensée. Ce contenu est le symbole vivant, par lequel se refont l'équilibre psychique et la progression de la libido. Comme l'explique Jung, la structure singulière du symbole unit les opposés:

De nature toujours infiniment complexe, le symbole se compose de données empruntées à toutes les fonctions psychiques; il n'est donc ni rationnel, ni irrationnel. D'une part accessible à la raison, il lui échappe d'autre

22. Jung, Mysterium, t. 2, p. 338.

23. Pour une recension des différents sens à donner au concept de Soi chez Jung, voir R. Gordon, "Tentative d'anatomie d'un concept: le Soi ", Cahiers jungiens de psychanalyse, 39 (1983) p. 52-61. 
part, puisqu'il est composé, à côté des données rationnelles, de celles, irrationnelles, qui viennent de la pure perception interne et externe. Par son côté divinatoire, par sa signification cachée, le symbole fait autant vibrer la pensée que le sentiment; sa singulière plasticité le revêt de formes sensoriellement perceptibles qui excitent la sensation autant que l'intuition $^{24}$.

La définition du symbole nous renseigne davantage sur l'aspect psychologique de l'expérience du Soi. Le symbole de totalité - le mandala - , au cœur de l'expérience de l'unus mundus, possède une structure quaternaire qui regroupe les quatre fonctions psychologiques. La vérité qui émane de cette expérience n'est pas seulement de l'ordre de la sensation et de la pensée, elle est aussi de l'ordre du sentiment et de l'intuition; elle se tient au carrefour des fonctions mentales, en faisant assumer à la conscience individuelle les paradoxes de l'existence humaine. Généralement, l'apparition du symbole unificateur fraye la voie à un apaisement salutaire, à une sérénité nouvelle qui raffermit l'âme par une assurance tranquille. Cet état de grâce est régulièrement décrit dans de nombreux textes de toutes les grandes religions, notamment sous forme de révélations. Lors d'un profond traumatisme, comme celui causé par la disparition d'un proche, la volonté se trouve affaiblie, comme écartelée entre des tonalités affectives contradictoires qui minent le libre arbitre de la personne affligée. Dans le cadre du processus de deuil, le symbole peut devenir le lieu d'expression d'un nouveau sens de la vie pour le sujet.

$\mathrm{Au}$ terme d'un cheminement qui conduit une personne vers l'individuation, la volonté reprend peu à peu le contrôle en établissant un équilibre qui manifeste un certain détachement face aux objets et situations qui rendaient la personne endeuillée prisonnière de ses émotions. Comme symptôme de la guérison, on retrouve chez cette personne une sérénité qui est à l'opposé des attitudes cambrées que l'on peut remarquer au cours des premières étapes du deuil. Les résistances typiques (comme le déni) que nous avons décrites plus haut comme appartenant à une personne en cheminement font alors place à une forme de détente beaucoup plus proche de la pente naturelle de la volonté telle que définie dans cet extrait par le physiologiste français Paul Chauchard:

24. Jung, Types psychologiques, p. 473. 
La volonté apparaît comme le contrôle cérébral de soi qui exige non de se mettre en état de tension mais, au contraire, dans cet optimum de vigilance harmonieux et disponible à base de calme et de paix intérieure. Cela permet d'être présent à ce qu'on pense et fait en étant un vrai ego humain, à base de schème corporel, dont l'association par conditionnement à tout processus cérébral est à la base de la volonté. Il s'agit de contrôler son imagination et de faire la synthèse de l'affectif et du raisonnable, à l'opposé des refoulements et défoulements ${ }^{25}$.

C'est donc à ce moment que, pour reprendre la définition de Freud, il est permis d'affirmer que la personne endeuillée a surmonté ses douleurs et s'est déprise de ses inhibitions. Le relâchement de la volonté témoigne de la fin de l'emprise du transfert sur la personne. La dépendance affective qu'entretenait cette dernière alors peut avoir été redirigée ou intériorisée afin de canaliser la libido vers un nouveau processus d'évolution, afin que la vie retrouve à nouveau son sens. À ce stade, et conformément au schéma de Kübler-Ross, la personne endeuillée manifeste une attitude de détachement face à la perte. Cela ne veut pas dire que la tristesse a fait place à l'indifférence, mais simplement que le sujet a pris une distance par rapport à ses émotions, ce qui lui permet désormais de vivre normalement, sans être prisonnier de sa peine.

\section{La mort et l'individuation dans les sociétés occidentales}

Malgré l'énorme souffrance qui l'accompagne, le deuil, ou plus généralement l'expérience de la mort, peut générer un équilibre psychique profond et durable, à condition bien sûr que le moi soit assez fort pour supporter cette épreuve. Nous avons vu qu'il existe de nombreuses possibilités de dérapage pour la personne qui subit ce genre de choc. L'expérience de la mort, de la souffrance et de l'impermanence des choses sont causes d'une nigredo psychologique qui initie tout processus d'individuation. Mais la route qui mène vers la renaissance et vers un nouvel équilibre est longue, et presque toujours très pénible. La confrontation avec l'inconscient demande une force et une persévérance qui ne sont pas acquises à toutes les personnalités. Cette difficulté n'est-elle pas redou-

25. P. Chauchard, "Volonté et contrôle cérébral», dans Science et conscience, Paris, Stock, 1980, p. 124. 
blée dans nos sociétés occidentales, où l'on ne tolère aucune forme de souffrance et où l'on cherche systématiquement le «vite fait »? Où ce qui s'inscrit dans la durée est rejeté comme inutile et trop abstrait? La rationalité instrumentale, nourrie d'une foi pragmatique inébranlable, avec pour credo "la fin justifie les moyens ", n'est-elle pas plutôt un facteur qui entrave toute expérience véritable de la finitude humaine?

Il ne faut pas oublier que les valeurs dominantes d'une société donnée sont déterminantes dans la constitution de la typologie psychologique des membres de cette société. Elles déterminent en bonne partie la structure de la persona ${ }^{26}$ puisque celle-ci est un facteur de relation avec le monde extérieur. On peut alors supposer que le choix de développement particulier d'une fonction psychologique spécifique est tributaire de la valeur accordée à cette fonction par la société pour l'adaptation à l'environnement. C'est pourquoi nous voulons nous interroger, en conclusion de notre exposé sur le deuil et l'individuation, sur l'incompatibilité apparente du pragmatisme, aujourd'hui dominant dans presque toutes les sociétés industrielles, et le développement de la capacité des individus à faire l'expérience de valeurs humaines fondamentales. Dans le contexte sociologique qui est le nôtre aujourd'hui, n'est-il pas en effet devenu de plus en plus difficile de promouvoir ces valeurs? Dans un univers technologique qui nous plonge dans une réalité virtuelle entièrement contrôlable, ne sommes-nous pas entraînés, comme par une espèce de conditionnement, à ne rechercher précisément que les situations que l'on peut manier à sa guise et à fuir celles qui, comme la mort, échappent à notre contrôle? Ne sommes-nous pas entraînés par conséquent vers une espèce d'infantilisme qui est bien loin de la maturité qu'exige au fond toute vie humaine authentique?

L'approche que les institutions occidentales adoptent par rapport au phénomène de la mort et du deuil nous semble dans bien des cas révélatrice de l'influence constante d'une rationalité instrumentale obsédée par le profit et l'efficacité. Pour illustrer cette situation, le sociologue suisse Jean Ziegler choisit les hôpitaux, qu'il considère comme des miroirs de nos valeurs pour les questions relatives à la mort: "L'hôpital

26. La persona correspond, chez Jung, au surmoi freudien. C'est une structure inconsciente qui modèle les choix moraux d'un individu à partir de l'influence de son éducation et de son environnement culturel. 
est un des microcosmes où se résument les conflits de la société marchande. D'une façon paroxystique, toutes ces lâchetés, toute l'inhumanité fondamentale de la rationalité du profit se concrétisent à l'hôpital, plus précisément au chevet de l'homme engagé dans le processus du mourir ${ }^{27}$. Dans le processus du mourir, la technologie médicale est devenue un ressort particulièrement efficace de la rationalité instrumentale, au point même où elle s'insère désormais entre la vie et la mort, et dépossède l'individu du choix de sa propre mort. Cette tendance de la rationalité instrumentale à vouloir se poser "comme maître et possesseur de la nature » caractérise fondamentalement les actions qu'elle entreprend: elle cherche le plus souvent à vouloir obstinément prolonger la vie en imposant une médicalisation du mourir qui est bien loin de l'intimité que requiert une telle expérience.

Il n'est pas très difficile de constater que les valeurs qui sous-tendent la vision occidentale du mourir, motivées par le souci obsessif d'efficacité et par le contrôle de la main humaine sur la nature, se retrouvent dans presque toutes les sphères d'activité de nos sociétés. Dans le domaine politique par exemple, presque tous les efforts des gouvernements, poussés il est vrai par la majorité de la population, se concentrent sur le bienêtre matériel des citoyens. Tout le discours public et médiatique est aujourd'hui pris en otage par le "monde du visible». Par l'économie, dont la réussite se mesure à l'augmentation du pouvoir d'achat des «consommateurs »; par la santé aussi, qui n'est évaluée qu'en fonction de la "qualité des soins ». Pourquoi en effet, croirait-on entendre, s'occuper de l'homme puisque de toute façon la machine et le pouvoir technologique le feront à notre place ? Pas étonnant dès lors que la machine, s'insérant de plus en plus lourdement entre l'homme et la nature, enveloppe celuici d'une réalité virtuelle qui au fond finit par l'enfermer dans le culte narcissique de lui-même. Nous sommes ici bien loin d'une culture dont le rôle premier serait d'élever l'homme en l'éveillant au réel, à sa beauté autant qu'à son aspect tragique.

Au fond, dans l'évolution des sociétés industrielles avancées, on peut remarquer que la dégradation du sentiment religieux a été suivie d'une croissance proportionnelle du rôle de l'État dans la vie des citoyens. L'autonomie qu'engendre une vie spirituelle riche, et soucieuse de situer

27. J. Ziegler, Les vivants et la mort, Paris, Seuil, 1975, p. 128. 
l'homme au sein d'une vision eschatologique qui donne un sens aux souffrances et aux malheurs qui l'accablent, a été au moins en partie abandonnée au pouvoir technique de l'État. De plus en plus, les citoyens en appellent à l'État pour régler leurs problèmes, même les plus personnels. L'omnipotence étatique qui en résulte ne peut qu'accroître la déresponsabilisation des individus de même que leur incapacité à trouver un équilibre personnel harmonieux. D'un autre côté, l'incapacité bien compréhensible de l'État à satisfaire la variété infinie des besoins du citoyen déresponsabilisé finit par discréditer le rôle des institutions démocratiques, avec pour conséquence le repli des individus sur des espaces publics qu'ils croient plus près de leurs préoccupations (quartiers, sectes, gangs, etc.).

Le processus jungien d'individuation nous enseigne que la survalorisation d'une fonction psychologique - ce qui est le cas pour une dynamique psychologique et culturelle privilégiant exclusivement la rationalité instrumentale - est antinomique par rapport à la possibilité de la réalisation du Soi. C'est ce que nous rappelle le thème de la mort, et c'est ce que nous aide à comprendre la psychanalyse. En mettant le Soi au centre du processus d'individuation, Jung sort la réflexion psychanalytique de la seule dialectique conscience-inconscient; elle insère l'homme au sein d'un plan plus vaste: celui de la destinée humaine. Par ce biais, la psychanalyse nous interpelle dans notre rapport à ce qui produit le sens dans nos vies, elle nous met en route vers Dieu, support ontologique du sens. Dès lors, la psychanalyse ouvre sur une pensée plurielle dans laquelle se tiennent aussi la théologie et la philosophie.

Dans cette quête multidisciplinaire, n'avons-nous donc pas la responsabilité, nous qui prétendons nous intéresser à l'âme humaine, de nous faire les apologistes les plus convaincus - et si possible les plus convaincants - de valeurs qui transcendent le visible et l'immédiat? Au-delà des disciplines et des confessions, n'y a-t-il pas pour nous tous un lieu de la réflexion qui nous convie à dépasser les barrières du présent sensible ? La psychanalyse, en particulier la psychanalyse jungienne, dans sa quête de l'équilibre personnel, nous a semblé en tout cas donner un poids et une légitimité à ceux qui, s'occupant des questions spirituelles, ne désespèrent pas de trouver pour l'homme un phare qui éclairera sa quête de sens. Ainsi peut-être, peu à peu, pourrons-nous acquérir la force nécessaire pour surmonter tous nos deuils et au moins la part essentielle des problèmes que nous cause la vie présente. 


\section{RÉSUMÉ}

Cet article entend aborder le thème du deuil et de la mort à travers l'approche du psychanalyste suisse Carl Gustav Jung. Ainsi, les deux premières parties du travail sont consacrées à la description et à la compréhension du processus de deuil, de même qu'aux étapes de la guérison psychologique nommée par Jung individuation. Par ce biais, nous cherchons à faire valoir la pertinence de la psychanalyse dans le champ philosophique, théologique et moral, tout en mesurant, dans la conclusion, les périls que font subir à la vie spirituelle de l'homme certains aspects de l'évolution culturelle de nos sociétés.

\section{ABSTRACT}

This article deals with grief and death through the works of the Swiss psychoanalyst Carl Gustav Jung. The first two sections describe and seek to understand grief and the different stages of psychological healing that Jung calls individuation. Through this, we hope to show the relevance of psychoanalysis in the realms of philosophy, theology and ethics. In our conclusion, we also measure how certain aspects of the cultural evolution of our societies endanger the human being's spiritual life.

(C) Revue Théologiques 2002. Tout droit réservé. 